Review Paper

\title{
WHAT THE SUNSPOT RECORD TELLS US ABOUT SPACE CLIMATE
}

\author{
DAVID H. HATHAWAY and ROBERT M. WILSON \\ NASA/Marshall Space Flight Center/NSSTC, Huntsville, AL 35812 \\ (e-mail:david.hathaway@msfc.nasa.gov)
}

(Received 1 September 2004; accepted 24 September 2004)

\begin{abstract}
The records concerning the number, sizes, and positions of sunspots provide a direct means of characterizing solar activity over nearly 400 years. Sunspot numbers are strongly correlated with modern measures of solar activity including: $10.7-\mathrm{cm}$ radio flux, total irradiance, X-ray flares, sunspot area, the baseline level of geomagnetic activity, and the flux of galactic cosmic rays. The Group Sunspot Number provides information on 27 sunspot cycles, far more than any of the modern measures of solar activity, and enough to provide important details about long-term variations in solar activity or "Space Climate." The sunspot record shows: 1) sunspot cycles have periods of $131 \pm 14$ months with a normal distribution; 2) sunspot cycles are asymmetric with a fast rise and slow decline; 3) the rise time from minimum to maximum decreases with cycle amplitude; 4) large amplitude cycles are preceded by short period cycles; 5) large amplitude cycles are preceded by high minima; 6) although the two hemispheres remain linked in phase, there are significant asymmetries in the activity in each hemisphere; 7) the rate at which the active latitudes drift toward the equator is anti-correlated with the cycle period; 8) the rate at which the active latitudes drift toward the equator is positively correlated with the amplitude of the cycle after the next; 9) there has been a significant secular increase in the amplitudes of the sunspot cycles since the end of the Maunder Minimum (1715); and 10) there is weak evidence for a quasi-periodic variation in the sunspot cycle amplitudes with a period of about 90 years. These characteristics indicate that the next solar cycle should have a maximum smoothed sunspot number of about $145 \pm 30$ in 2010 while the following cycle should have a maximum of about $70 \pm 30$ in 2023.
\end{abstract}

\section{Introduction}

Understanding the solar activity cycle remains as one of the key problems in solar physics. Solar activity impacts us in many ways. It poses hazards to our satellites in space, our technology on the ground, and even ourselves as we venture into space. Solar activity has well-known impacts on Earth's magnetosphere and ionosphere. There is also mounting evidence that solar activity has an influence on terrestrial climate (Eddy, 1976; Reid, 1987; Friis-Christensen and Lassen, 1991; Lean, Beer, and Bradley, 1995; Hoyt and Schatten, 1997). The significance of these societal and natural impacts makes it all the more important that we understand how and why solar activity occurs. Understanding why it occurs is the goal of solar dynamo theory. Understanding how it occurs is the goal of many of our solar observations, from visual observations of sunspots to helioseismic observations of fluid flows in the solar interior. Here we review the visual observations of sunspots to determine 
what these observations tell us about how the Sun varies on time scales from years to centuries.

\section{Sunspot Observations}

Sunspots are the most easily observed solar features that are related to solar activity. While occasional mention of naked-eye sunspots can be found in historical records prior to 1610, it was Galileo's telescopic observations of sunspots in that year that started detailed and systematic observations of sunspots (Bray and Loughhead, 1964). The 11-year variation in the number of sunspots was first noted by Schwabe (1844). Shortly thereafter (1848) Rudolf Wolf at the Swiss Federal Observatory in Zürich, Switzerland devised his measure of sunspot numbers that continues to this day as the International Sunspot Number (Kiepenheuer, 1953; Waldmeier, 1961; McKinnon, 1987). Wolf recognized that it is far easier to identify sunspot groups than it is to identify each individual sunspot. His "relative" sunspot number, $R_{Z}$, thus emphasized sunspot groups with

$$
R_{Z}=k(10 g+n),
$$

where $k$ is a correction factor for the observer, $g$ is the number of identified sunspot groups, and $n$ is the number of individual sunspots. These Zurich or International Sunspot Numbers have been obtained daily since 1848 . Wolf extended the data back to 1749 using older records but much of that earlier data is incomplete.

Recently Hoyt and Schatten (1998a,b) devised a sunspot number index based solely on the number of observed sunspot groups. This index, $R_{G}$, is devised to give similar numbers to the International Sunspot Number by introducing a multiplier so that

$$
R_{G}=\frac{1}{N} \sum_{i=1}^{N} k_{i} 12.08 g_{i},
$$

where $N$ is the number of observers, $k_{i}$ is the correction factor for observer $i$, and $g_{i}$ is the number of sunspot groups reported by observer $i$. Hoyt and Schatten have examined thousands of additional historical records to augment the data examined by Wolf and to extend the dataset back to Galileo's observations in 1610. Although some gaps are still present, this Group Sunspot Number gives a more complete and longer dataset than the International Sunspot Number.

Sunspot number indices are good indicators of solar activity but additional information about how the Sun varies is contained in data concerning the sizes and positions of the sunspots. The Royal Greenwich Observatory has recorded this information using observations from affiliated observatories starting in March of 1874 and ending in 1976. The photographic plates obtained at Mt. Wilson Observatory from 1917 to 1982 were measured by Howard, Gilman, and Gilman (1984) to give individual sunspot areas and positions. Areas and positions of sunspot groups 
have been reported by the US National Oceanic and Atmospheric Administration from 1976 to the present. Together these datasets (and similar datasets from other observatories) provide continuous daily observations from 1874 to the present.

\section{Sunspots and Solar Activity}

Modern observations of the Sun provide a vast array of measures of solar activity. These observations span the electromagnetic spectrum from radio waves to gamma-rays and include particles and plasma waves from the Sun. In addition, we can measure magnetic fields on the surface of the Sun and flow fields on the surface and in the interior. Many of these observations are more directly related to the sources of solar activity. Because of this, some researchers discredit sunspot numbers as archaic and of little value. However, the sunspot numbers track these modern measures of solar activity very well. More importantly, the sunspot numbers have the distinct advantage of covering more than 27 sunspot cycles.

Figure 1 shows several modern indices plotted as functions of smoothed Group Sunspot Number. This plot shows that $R_{G}$ is strongly correlated with the sunspot area (Figure 1a), the $10.7 \mathrm{~cm}$ radio flux (Figure 1b), the monthly number of GOES
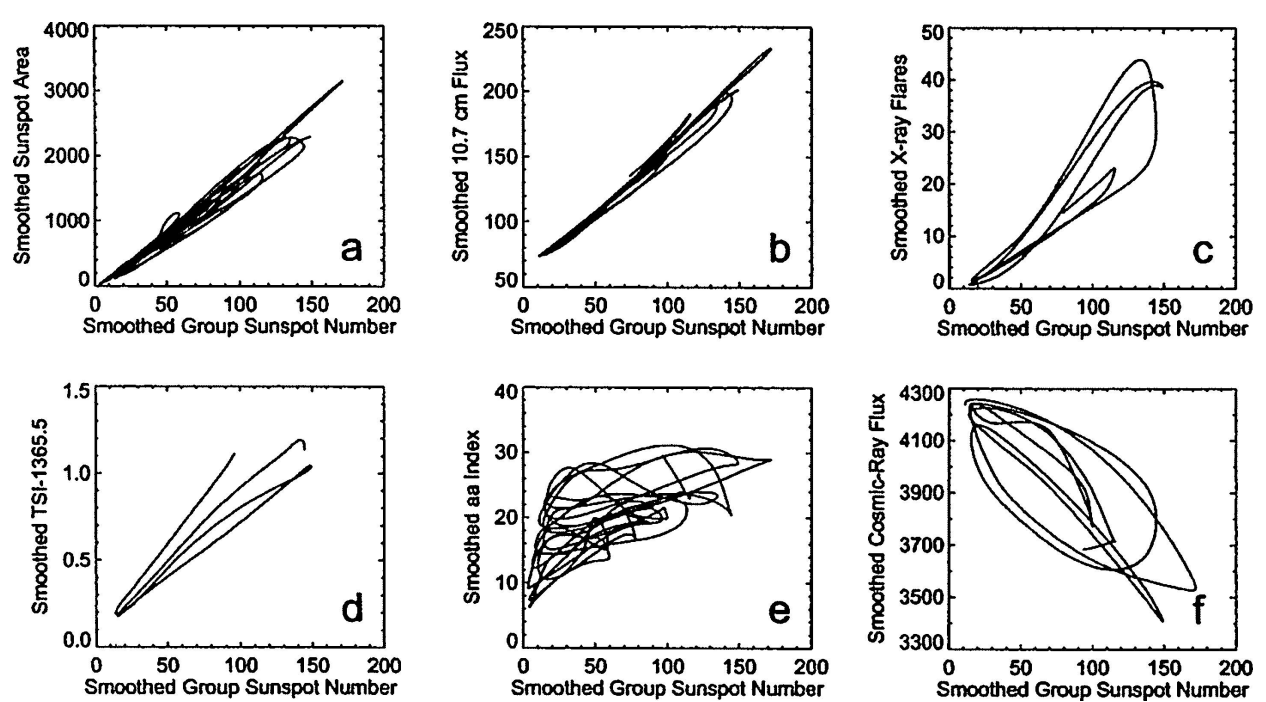

Figure 1. Modern indices of solar activity as functions of smoothed Group Sunspot Number. The smoothed sunspot area plotted in 1a has a $99 \%$ correlation with $R_{G}$. The smoothed $10.7 \mathrm{~cm}$ radio flux plotted in $1 \mathrm{~b}$ also has a $99 \%$ correlation with $R_{G}$. The smoothed monthly number of X-and M-class flares plotted in 1c has a 93\% correlation with $R_{G}$. The smoothed Total Solar Irradiance plotted in $1 \mathrm{~d}$ has a $96 \%$ correlation with $R_{G}$. The smoothed geomagnetic $a a$-index plotted in 1e has a baseline (minimum) value that has a $91 \%$ correlation with $R_{G}$. The smoothed cosmic ray flux from the Climax neutron monitor plotted in $1 \mathrm{f}$ has an $84 \%$ anti-correlation with $R_{G}$. This anti-correlation increases to $91 \%$ when an 8-month time lag with $R_{G}$ is included. 
$\mathrm{X}$-ray flares of classes $\mathrm{M}$ and $\mathrm{X}$ (Figure 1c), the total solar irradiance from the Physikalisch-Meteorologisches Observatorium Davos composite (Figure 1d), and the base level of geomagnetic activity as indicated by the aa index (Figure 1e). This figure also shows that $R_{G}$ is anti-correlated with the galactic cosmic-ray flux (Figure 1f). [Note that the smoothing employed here is the 24-month tapered Gaussian discussed in Hathaway, Wilson, and Reichmann (2002) and in the following section.] Flares, geomagnetic activity, and cosmic ray flux all exhibit time delays with activity lagging behind sunspot number. Stronger correlations can be obtained by including these time lags.

\section{Individual Sunspot Cycle Characteristics}

Sunspot numbers, areas, and positions have all been used to characterize the sunspot cycle. Even fundamental characteristics such as period and amplitude are, however, dependent upon how the data is filtered and sampled. Our single point-of-view from Earth allows us to observe only one hemisphere so all measures of solar activity are hemispheric and not global in nature. The rotation of the Sun allows us to see the entire surface but only after the passage of at least half of its 27 day rotation, during which solar activity can change significantly. The only solution is to average quantities such as sunspot number over at least a solar rotation. The standard solution is to use monthly averages and then average these over a 13month interval centered on a given month. This 13-month running mean, even with its tapered ends, allows many high frequencies to pass. Hathaway, Wilson, and Reichmann (2002) have discussed this and show how a tapered Gaussian shaped average reduces this problem. The resulting smoothed signals do not have the many multiple maxima and minima that can give ambiguous values to both the size and timing of a cycle maximum or minimum. In this section we will use the 24-month tapered Gaussian to smooth the data before we determine the value and epoch of the various maxima and minima.

The 11-year period of the sunspot cycle was first noted by Schwabe (1844) from just 17 years of sunspot data. Using the 27 cycles covered by the Group Sunspot number leaves this relatively unchanged. Hathaway, Wilson, and Reichmann (2002) found an average period of $131 \pm 14$ months from minimum-to-minimum with a distribution that cannot be distinguished from a normal (Gaussian) distribution. This result is shown in Figure 2. This is in contrast to the bimodal distributions discussed by Wilson (1987) and by Rabin, Wilson, and Moore (1986). The difference can be attributed primarily to the different smoothing functions used and secondarily to the use of Group rather than the International Sunspot Number. Cycle length determinations using other indicators of solar activity and significantly different methods also give similar results (Fligge, Solanki, and Beer, 1999).

The systematic asymmetry of the sunspot cycle was first noted by Waldmeier $(1935,1939)$. The time from minimum to the following maximum is usually shorter 

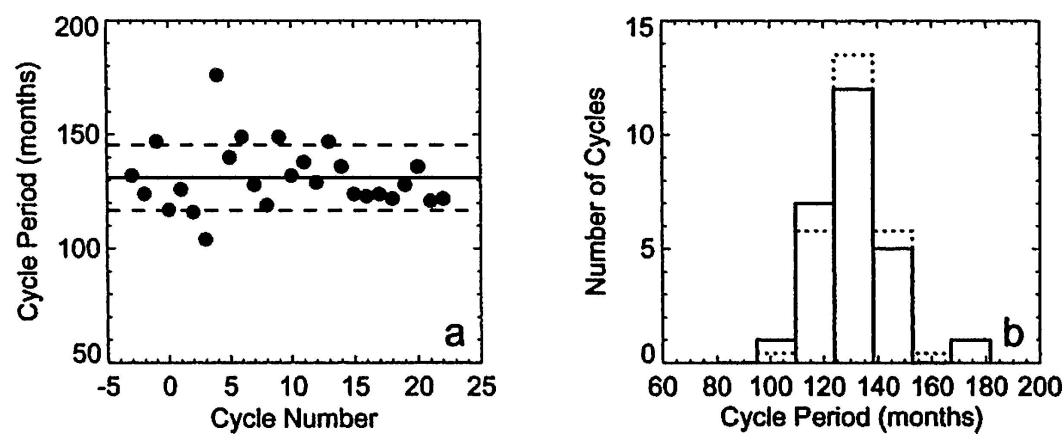

Figure 2. Cycle period distributions. The sunspot cycle period as a function of time (cycle number) is shown in panel a. A histogram of the cycle periods in 1-standard deviation (14-month) bins about the average (131.1 months) is shown by the solid line in panel $\mathrm{b}$. The dotted line in panel b shows the normal distribution.

(76\%) than the time from that maximum to the following minimum. The length of the rise time is inversely related to the amplitude of the cycle. This "Waldmeier Effect" is evident in the smoothed Group Sunspot Numbers as shown in Figure 3 using data from 1797 to the present (earlier data was less complete). The anticorrelation between the rise-times and amplitudes of the cycles is weak $(-0.35)$ but significant.

There is also a significant correlation $(0.8)$ between the sunspot number at minimum and at maximum as well as an anti-correlation $(-0.6)$ between the amplitude of a cycle and the period of the previous cycle. These relations were noted by Hathaway, Wilson, and Reichmann $(1999,2002)$ and are shown in Figure 4. Solanki et al. (2002) found similar behavior in the sense that the anti-correlation between cycle amplitude and cycle length has a lag with large amplitudes coming after
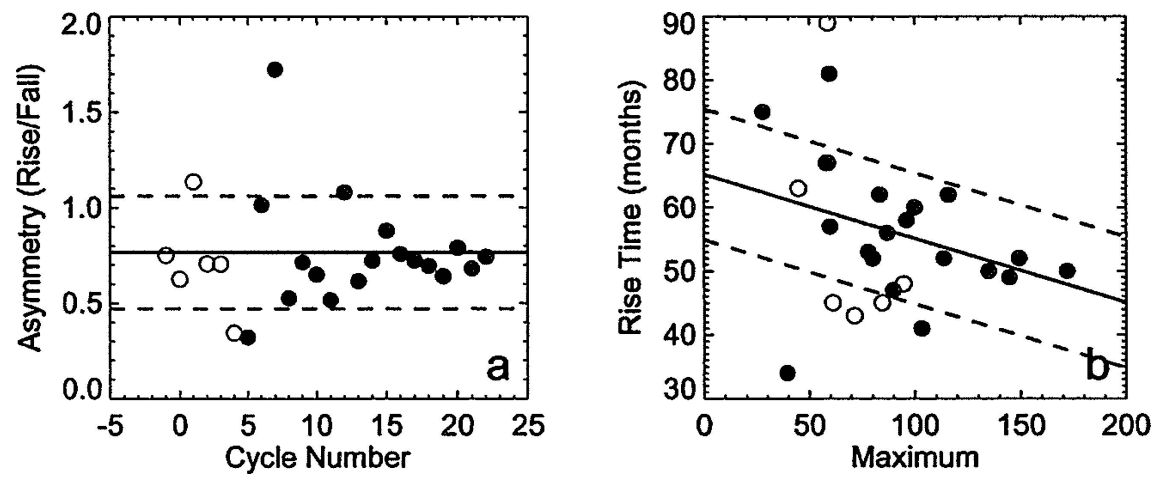

Figure 3. Cycle shape asymmetry. The ratio of the cycle rise time to the cycle fall time as a function of cycle number since 1797 is shown in panel a by the filled circles. Earlier data are shown with open circles. The decrease in rise time with cycle amplitude (the Waldmeier Effect) is shown in panel b. Large amplitude cycles take less time to rise to maximum. 

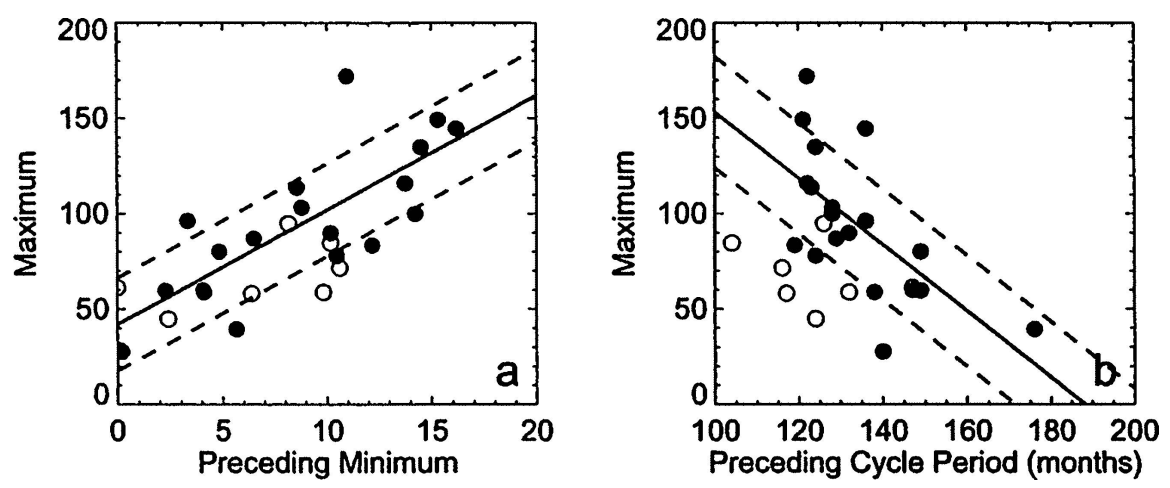

Figure 4. Correlation between cycle maximum and minimum (panel a) and between cycle maximum and the period of the previous cycle (panel b). Data from 1797 on are shown with filled circles. Earlier data are shown with open circles. Large amplitude cycles follow short cycles and high minima.

short cycles. The anti-correlation between the amplitude and period of the previous cycle is somewhat skewed by the point representing the small cycle 5 following the long cycle 4 . We should note that the length of cycle 4 has been called into question. Usoskin, Mursula, and Kovaltsov $(2001,2002)$ suggest that cycle 4 actually consists of two short period cycles; one from 1784 to 1793 and another from 1793 to 1800 . This would replace the one extremely long period cycle in Figure 2b with two extremely short period cycles. It would also weaken the anti-correlation between rise-time and amplitude seen in the Waldmeier effect (Figure 3b) by introducing a small but rapidly rising additional cycle and significantly alter the relationship between amplitude and previous cycle period (Figure 4b) by replacing the small cycle following the longest cycle with two small cycles following each of the two shortest cycles. While Krivova, Solanki, and Beer (2002) find little evidence for this "lost cycle" in the sunspot number statistics, the cosmogenic radioisotope records, or the auroral records, Usoskin, Mursula, and Kovaltsov (2003) maintain that the sunspot statistics are consistent with the existence of this "lost cycle."

The equatorward drift of the sunspot zones was first noted by Carrington (1858) but has been attributed to Spörer by Maunder (1890) and others. The effect is shown graphically in the famous "Butterfly Diagram" of Maunder (1904). Recently Hathaway et al. $(2003,2004)$ examined this equatorward drift by separating the cycles and finding the centroid position of the sunspot area in each hemisphere. The centroid of the sunspot area starts each cycle at mid-latitudes and drifts rapidly toward the equator. The drift rate slows and comes to a halt as the centroid approaches a latitude of about $8^{\circ}$. They find that the drift rate at the time of cycle maximum is anti-correlated with the period of the cycle and positively correlated with the amplitude of the second following $(N+2)$ cycle. These relations are shown in Figure 5. 

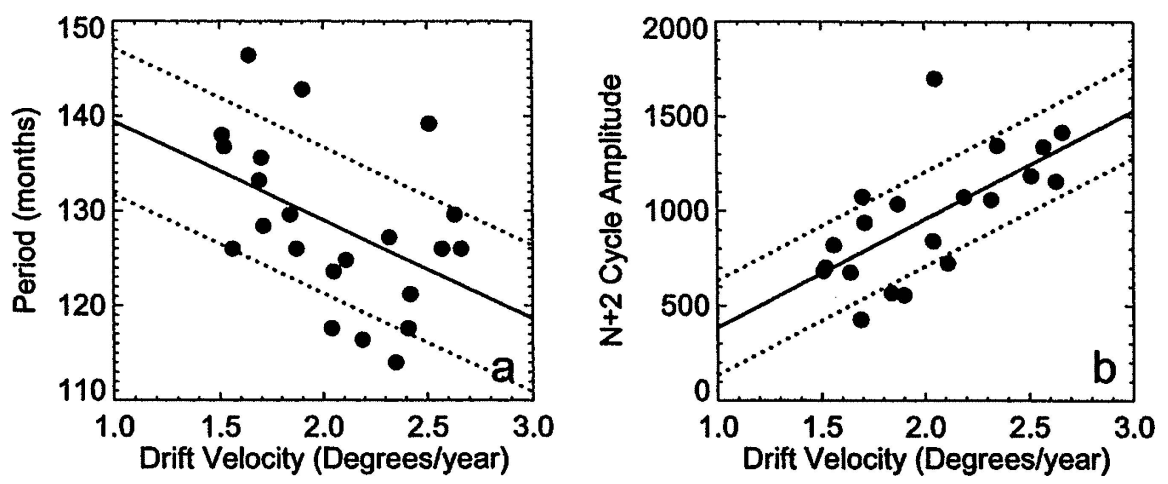

Figure 5. Cycle period and $N+2$ cycle amplitude as functions of the drift rate of the sunspot area centroid at cycle maximum. The cycle period (panel a) has a significant anti-correlation $(-0.5)$ with the drift velocity at maximum. The amplitude (in sunspot area) of the $N+2$ (second following) cycle (panel b) has a significant positive correlation (0.7) with the drift velocity at maximum.

These relationships accord well with dynamo models that include the effects of a deep meridional flow (Dikpati and Charbonneau, 1999; Nandy and Choudhuri, 2002). They also provide early estimates for the period of the current cycle and the amplitudes of the next two cycles. The drift velocities during the previous cycle (Cycle 22) were 2.4 degrees/year in both the north and south. This indicates amplitudes of $1200 \mu \mathrm{hem}$ in each hemisphere or a total sunspot number (from Figure 1a) of about $145 \pm 30$ for Cycle 24. The drift velocities for the current cycle (Cycle 23) are 1.4 degrees/year in the north and 0.9 degrees/year in the south. These values are both the lowest on record. Using the relationship from the previous cycles give an amplitude of $600 \mu \mathrm{hem}$ in the north and $300 \mu \mathrm{hem}$ in the south or a total sunspot number of about $70 \pm 30$ for Cycle 25 . These slow drift velocities should also give a long period of about $138 \pm 7$ months for the current cycle.

\section{North-South Asymmetry}

Solar activity shows variations between northern and southern hemispheres. In Figure 6a the monthly average sunspot areas in the northern hemisphere are plotted as positive values while the sunspot areas in the southern hemisphere are plotted as negative values. The hemispheres remain largely in phase but show asymmetries. Carbonell, Oliver, and Ballester (1993) have shown that these asymmetry variations are both real and significant in that they are not the result of random fluctuations. Figure $6 \mathrm{~b}$ shows the asymmetry (north-south) in sunspot area. There are significant swings from one hemisphere to the other with long intervals during which one hemisphere dominates. Figure 6c shows more recent data compared with data for flares, sunspot group numbers, and magnetic flux. All four indices 

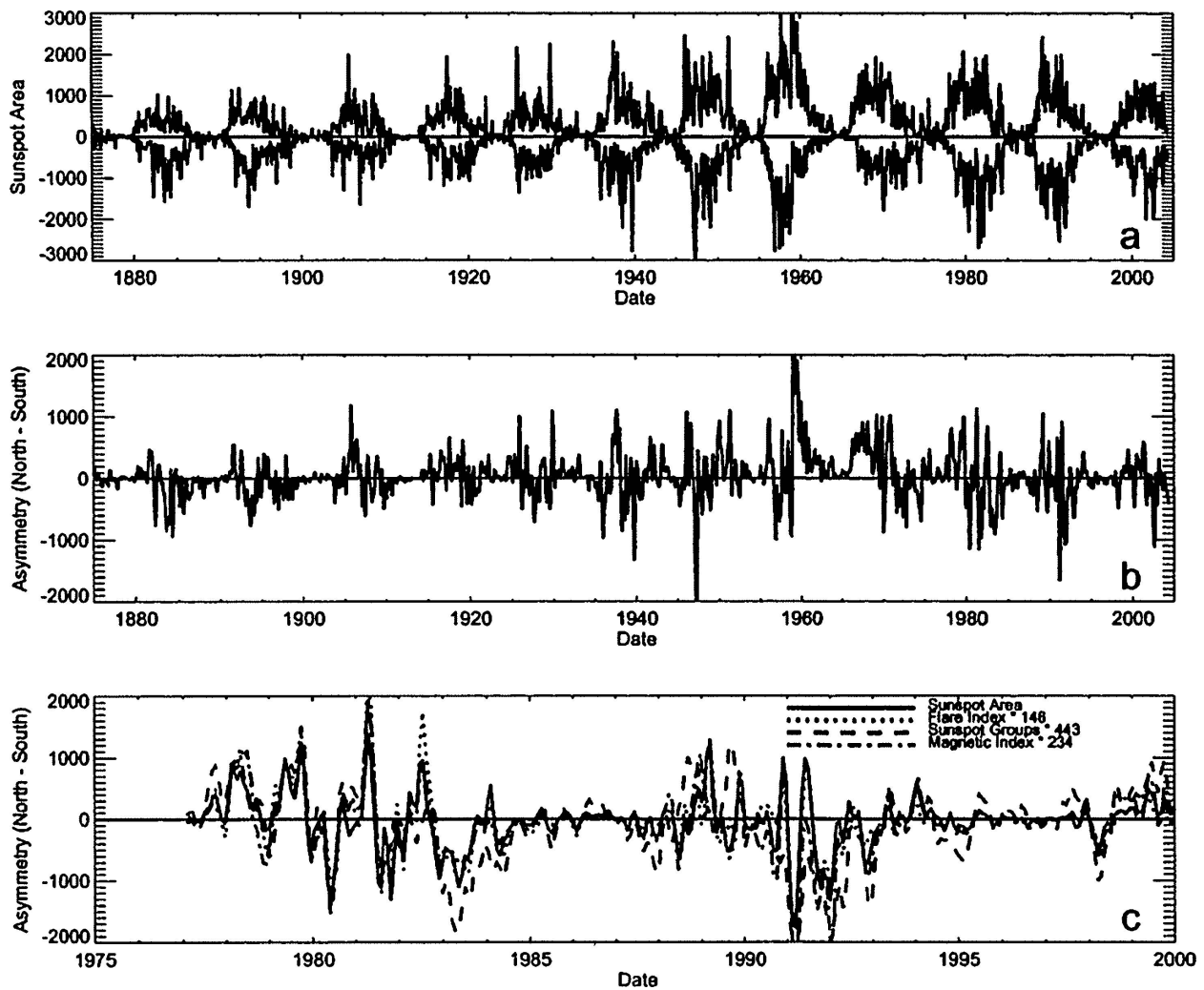

Figure 6. North-south asymmetry in solar activity. Panel a shows sunspot area in the north as positive values and sunspot area in the south as negative values. Panel $b$ shows the asymmetry in sunspot area in millionths of a hemisphere since 1875. Panel c shows more recent data compared with a flare index, the number of sunspot groups, and a magnetic index. The north-south asymmetries are well correlated with different indices and persist for significant periods of time but the hemispheres remain largely in phase.

show well-correlated north-south asymmetries. There are some indications of periodicities in the asymmetry (Ataç and Özgüç, 1996; Javaraiah and Gokhale, 1997), but their significance and connections to processes that drive solar activity remain uncertain.

\section{Active Longitudes}

Sunspots and solar activity also appear to cluster in "active longitudes." Bumba and Howard (1965) and Sawyer (1968) noted that new active regions grow in areas previously occupied by old active regions. Bogart (1982) found that this results in a periodic signal that is evident in the sunspot number record. 

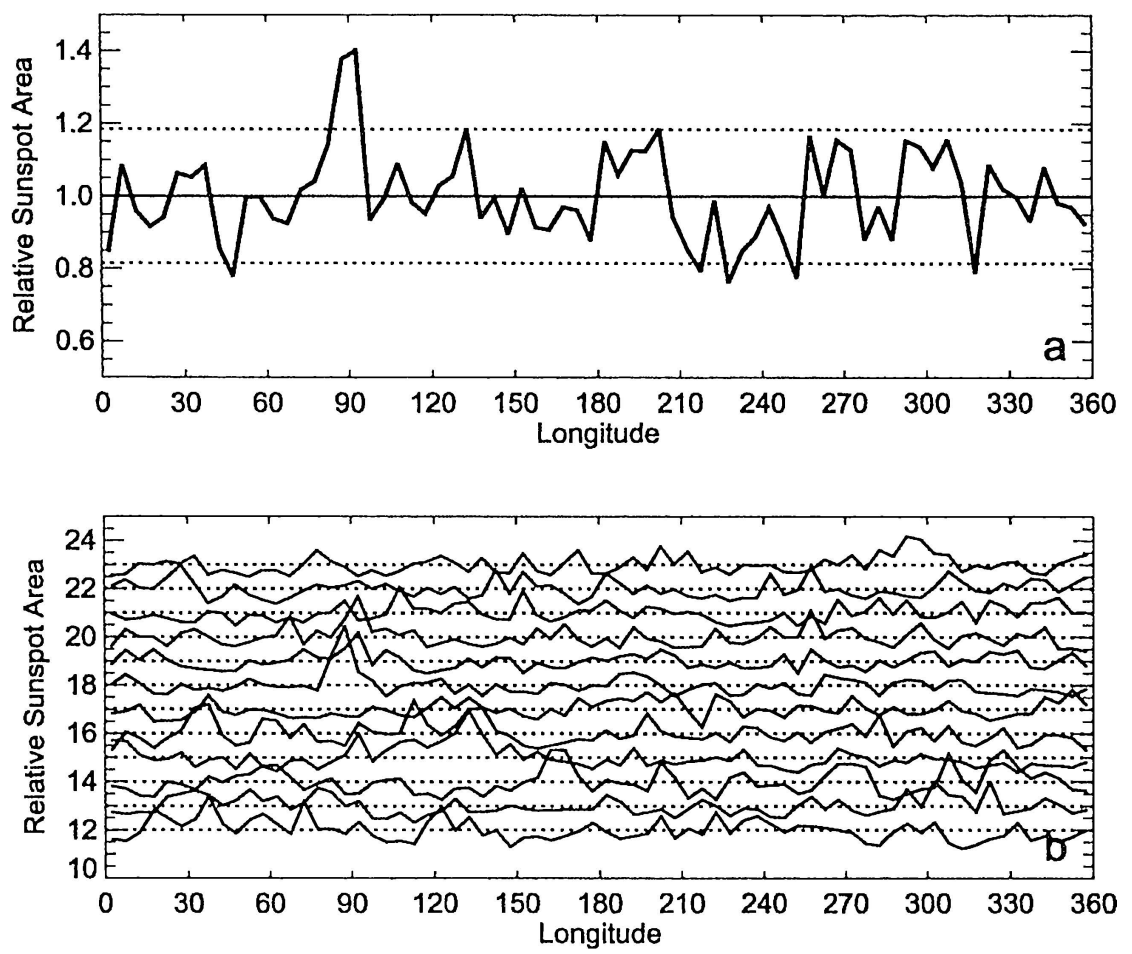

Figure 7. Active longitudes in sunspot area. The normalized sunspot area in $5^{\circ}$ longitude bins is plotted in panel a for the years 1878-2004. The dotted lines represent two standard errors in the normalized values. The sunspot area in several longitude bins meets or exceeds these limits. The individual cycles (12 through 23) are shown in panel $b$ with the normalized values offset in the vertical by the cycle number. Some active longitudes appear to persist from cycle to cycle.

Figure 7 illustrates the active longitude phenomena. In Figure 7 a the sunspot area in $5^{\circ}$ longitude bins averaged over 1682 solar rotations since 1878 and normalized to the average value per bin is plotted as a function of Carrington longitude. The $2 \sigma$ uncertainty in these values is represented by the dotted lines. This $2 \sigma$ limit is reached at several longitudes and significantly exceeded at two $\left(85-90^{\circ}\right.$ and $\left.90-95^{\circ}\right)$. Figure $7 \mathrm{~b}$ shows similar data for each individual cycle with the normalized value offset in the vertical by the sunspot cycle number. There are many peaks at twice the normal value and one, in cycle 18 at $85-90^{\circ}$, at three times the normal value. Some of these peaks even appear to persist from one cycle to the next, a result that has been noted by many authors including Bumba and Henja (1991), Mikhailutsa and Makarova (1994), and Bai (2003). Berdyugina and Usoskin (2003) find that the effect is more noticeable with hemispheric data. They find evidence for two active longitudes separated by $180^{\circ}$ that alternate in dominance and drift relative to the Carrington longitudes. The relationships these structures and their magnetic counterparts have with solar dynamo processes have been explored recently in a 
dynamo model by Moss (2004) who shows that oscillating multipole components can reproduce these effects.

\section{Short-Term Variability}

There are significant variations in solar activity on time scales shorter than the sunspot cycle. This is evident when the sunspot number record is filtered to remove both solar rotation effects (periods of about 27 days and less) and solar cycle effects. This signal is shown in Figure 8 for the last 50 years. In this figure the daily sunspot numbers are filtered with a tapered Gaussian shaped filter with a FWHM of 54 days. This reduces all signals with periods shorter than 54 days to less than $2 \%$ of their original amplitude. The resulting signal is sampled at 27 day intervals and then filtered again with a similar Gaussian with a FWHM of 24 rotations. Figure $8 \mathrm{~b}$ shows this final signal for the last 50 years while Figure 8a shows the residual obtained when this smoothed sunspot number signal is subtracted from
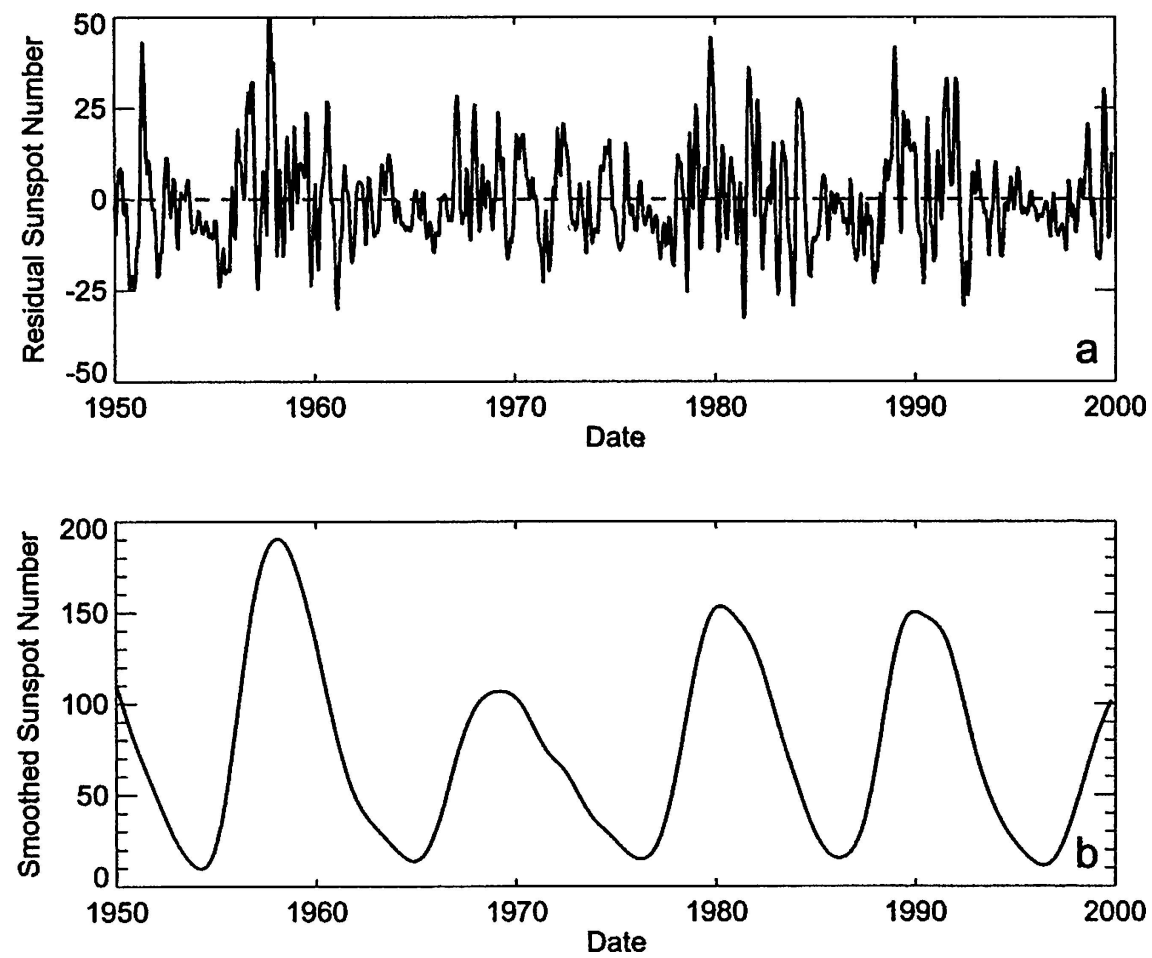

Figure 8. Residual sunspot numbers showing short-term variations. Panel $\mathrm{b}$ shows the smoothed sunspot number with its solar cycle behavior. Panel a shows the residual signal obtained when the smoothed behavior is subtracted from the monthly ( 27 day) data. The residual sunspot numbers appear rather chaotic but with episodes of periodic variations. 
54 day filtered data. This residual signal is quite chaotic but shows some interesting behavior.

A number of periodicities have been identified in various solar activity indices. In particular a 155-day periodic signal was noted in solar flare activity seen from SMM by Rieger $e t$ al. (1984). This signal was found in flares during other cycles by Bai and Cliver (1990) as well as in photospheric magnetic flux (Ballester, Oliver, and Carbonell, 2002). Lean (1990) analyzed the signal in the sunspot area data and found that it occurs in episodes and that its frequency drifts as well.

Another interesting periodicity is one found with a period of about 2 years (Waldmeier, 1973; Benevolenskaya, 1996; Mursula, Zieger, and Vilppola, 2003). This signal is particularly interesting because it helps to explain why some sunspot cycles exhibit double peaks and it may have its source in the oscillations seen at the base of the convection zone (Howe et al., 2000).

\section{Long-Term Variability}

Since the Maunder Minimum there has been a steady increase in sunspot cycle amplitudes (Wilson, 1988). This is readily seen in the smoothed Group Sunspot Numbers plotted in Figure 9. The thick lines in Figure 9 show the linear trend and the 1-sigma limits. Hathaway, Wilson, and Reichmann (2002) find a correlation coefficient of 0.7 between cycle amplitude and cycle number. The linear trend with time shown in Figure 9 reduces the variance in the cycle amplitudes from 36.5 to 24.1.

Numerous authors have noted multicycle periodicities in the sunspot cycle amplitudes. Gleissberg (1939) noted a periodicity of seven or eight cycles in cycle amplitudes from 1750 to 1928. While Garcia and Mouradian (1998) suggest that

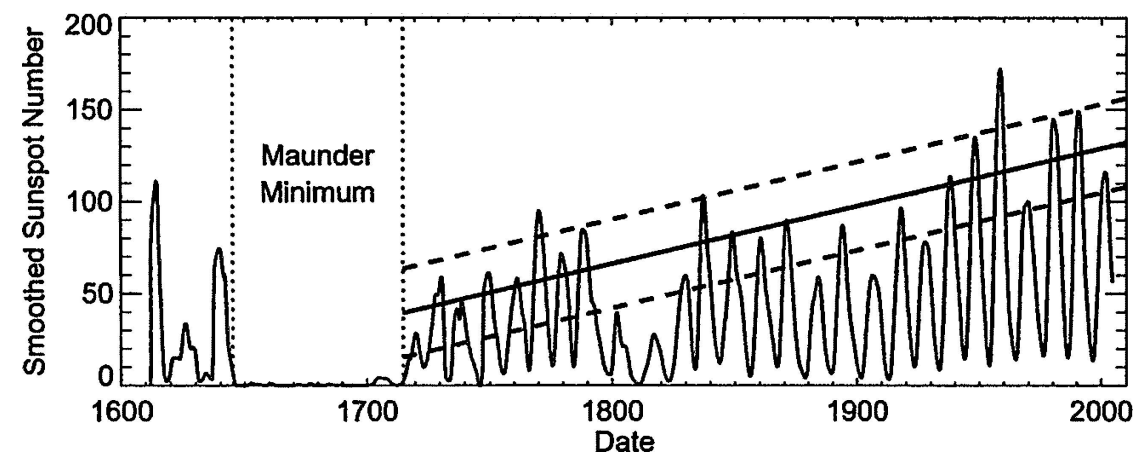

Figure 9. Linear increase in cycle amplitudes since the Maunder Minimum. The smoothed Group Sunspot Numbers are shown with the thin curve. The linear trend since the Maunder Minimum is shown with the thick line while the 1-sigma limits are shown with the dashed lines. This linear trend is the most significant long-term variation in the sunspot cycle amplitudes. 
a third period of this cycle can be found in the sunspot data, others (Hathaway, Wilson, and Reichmann, 1999) suggest that the period is changing or (Rozelot, 1994; Ogurtsov et al., 2002) that it consists of two different components (one with a 90-100 year period and a second with a 50-60 year period). A simple sinusoid fit to the residual cycle amplitudes when the secular trend is removed gives a 9.1 cycle periodicity that reduces the variance from 24.1 to 20.8 .

Periodicities of two cycles (Gnevyshev and Ohl, 1948; Wilson, 1986; Mursula, Usoskin, and Kovaltsov, 2001) and three cycles (Ahluwalia, 1998) have also been suggested. The best fit to a 3-cycle periodicity gives an insignificant reduction in the standard deviation (from 24.1 to 23.6) and an insignificant correlation coefficient (0.19). Similarly, the best fit to a 2-cycle periodicity reduces the standard deviation from 24.1 to 23.7 and has a correlation coefficient of only 0.18 .

\section{Conclusions}

The record of sunspot numbers, positions, and areas provide significant information for characterizing the sunspot cycle and its long-term variability. The sunspot cycles have periods of about $131 \pm 14$ months with rise times that are inversely proportional to their amplitudes. High maxima follow short cycles and high minima. There are significant asymmetries between the northern and southern hemispheres that often persist for years. We have seen a steady increase in the amplitudes of the cycles since the Maunder Minimum and find some evidence for long-term periodicity on a time-scale of about 90 years.

Some of these characteristics can be combined with parametric curves to provide predictions of the level of activity throughout a solar cycle from a time well before maximum (Hathaway, Wilson, and Reichmann, 1994). The drift rates of the centroid of sunspot area in each hemisphere (Hathaway et al., 2003, 2004) provide early estimates for both the timing and amplitude of future cycles (Section 4 and Figure 5). These characteristics can be combined to give an estimate of solar activity more than 20 years into the future. One problem that arises is a conflict concerning the estimate for the length of the current cycle. The anti-correlation between drift rate and cycle period (Figure 6a) indicates a long period (138 months) for Cycle 23 based on the slow drift rates. However, the high drift rates during Cycle 22 indicate (Figure 5b) that the next cycle will be large $\left(R_{G} \sim 145 \pm 30\right)$ so, according to the results shown in Figure 4b, the period of Cycle 23 should be short, about 110 months. Since the anti-correlation between period and amplitude of the following cycle is slightly stronger $(-0.6)$ than the anti-correlation between period and drift rate $(-0.5)$ a period of about 120 months is more appropriate. This gives the next minimum in 2006. The small amplitude $\left(R_{G} \sim 70 \pm 30\right)$ expected for Cycle 25 indicates (Figure 4b) that Cycle 24 should have a long period of about 155 months. This gives a minimum for Cycle 25 in 2019. These amplitudes and times of minima give predicted activity levels shown in Figure 10. 


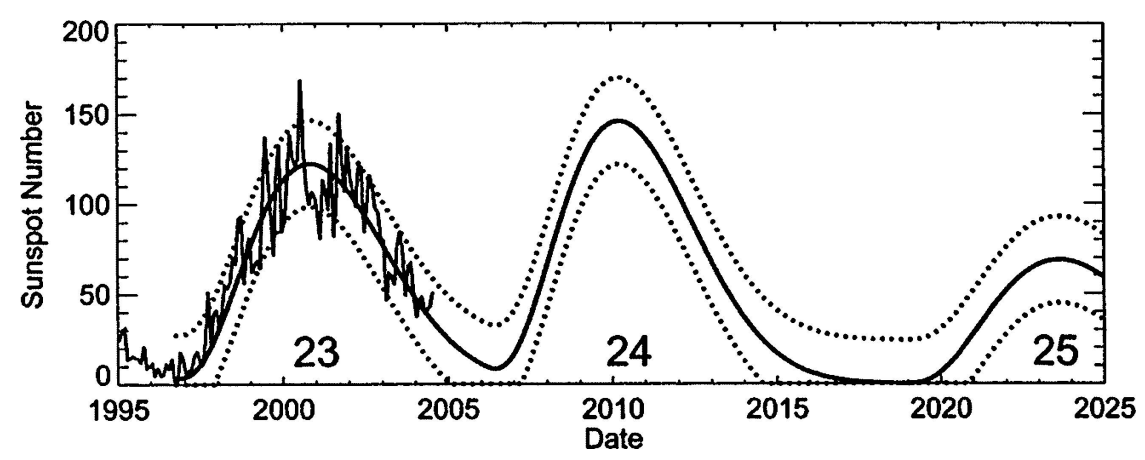

Figure 10. Predicted sunspot numbers using sunspot cycle characteristics. Predicted values are shown by the thick smooth curve. The dotted curves indicate the expected range of variation. The jagged line shows the monthly averaged International Sunspot Numbers. The drift rate of the sunspot area centroids indicate a large cycle for Cycle 24 and a small cycle for Cycle 25. These amplitudes in turn indicate a short cycle for Cycle 23 and a long cycle for Cycle 24.

\section{Acknowledgments}

This work was supported by a grant from the Supporting Research and Technology program in NASA's Office of Space Science through its SunEarth Connection Enterprise. Sunspot number data was provided by SIDC, RWC Belgium, World Data Center for the Sunspot Index, Royal Observatory of Belgium at http://sidc.oma.be/index.php3, and by Douglas Hoyt and Kenneth Schatten at ftp://ftp.ngdc.noaa.gov/STP/SOLAR DATA/. Sunspot areas were provided by the Royal Greenwich Observatory, the US Air Force, and the National Oceanic and Atmospheric Administration and are available at http://science.nasa.gov/ssl/pad/solar/greenwch.htm. The composite total solar irradiance data came from the World Radiation Center, Physikalisch-Meteorologisches Observatorium Davos. Data on X-ray flares and geomagnetic variations were provided by NOAA's Space Environment Center. Data on the $10.7 \mathrm{~cm}$ radio flux came from the Canadian National Research Council's Dominion Radio Astrophysical Observatory. Data on the galactic cosmic rays came from the Climax Neutron Monitor. We are gratefully indebted to these individuals and institutions and their funding organizations.

\section{References}

Ahluwalia, H. S.: 1998, J. Geophys. Res. 103, 12, 103.

Ataç, T. and Özgüç, A.: 1996, Solar Phys. 166, 201.

Bai, T.: 2003, Astrophys. J. 585, 1114.

Bai, T. and Cliver, E. W.: 1990, Astrophys. J. 363, 299.

Ballester, J. L., Oliver, R., and Carbonell, M.: 2002, Astrophys. J. 566, 505. 
Benevolenskaya, E. E.: 1996, Solar Phys. 167, 47.

Berdyugina, S. V. and Usoskin, I. G.: 2003, Astron. Astrophys. 405, 1121.

Bogart, R. S.: 1982, Solar Phys. 76, 155.

Bray, R. J. and Loughhead, R. E.: 1964, Sunspots, John Wiley \& Sons, New York, 303 pp.

Bumba, V. and Henja, L.: 1991, Bull. Astron. Inst. Czechosl. 42, 76.

Bumba, V. and Howard, R.: 1965, Astrophys. J. 141, 1502.

Carbonell, M., Oliver, R., and Ballester, J. L.: 1993, Astron. Astrophys. 274, 497.

Carrington, R. C.: 1858, Mon. Not. R. Astron. Soc. 19, 1.

Dikpati, M. and Charbonneau, P.: 1999, Astrophys. J. 518, 508.

Eddy, J. A.: 1976, Science 192, 1189.

Fligge, M., Solanki, S. K., and Beer, J.: 1999, Astron. Astrophys. 346, 313.

Friis-Christensen, E. and Lassen, K.: 1991, Science 254, 698.

Garcia, A. and Mouradian, Z.: 1998, Solar Phys. 180, 495.

Gleissberg, M. N.: 1939, Observatory 62, 158.

Gnevyshev, M. N. and Ohl, A. I.: 1948, Astron. Zh. 25, 13.

Hathaway, D. H., Wilson, R. M., and Reichmann, E. J.: 1994, Solar Phys. 151, 177.

Hathaway, D. H., Wilson, R. M., and Reichmann, E. J.: 1999, J. Geophys. Res. 104, 22,375.

Hathaway, D. H., Wilson, R. M., and Reichmann, E. J.: 2002, Solar Phys. 211, 357.

Hathaway, D. H., Nandy, D., Wilson, R. M., and Reichmann, E. J.: 2003, Astrophys. J. 589, 665.

Hathaway, D. H., Nandy, D., Wilson, R. M., and Reichmann, E. J.: 2004, Astrophys. J. 602, 543.

Howard, R. F., Gilman, P. A., and Gilman, P.: 1984, Astrophys. J. 283, 373.

Howe, R., Christensen-Dalsgaard, J., Hill, F., Komm, R. W., Larsen, R. M., Schou, J., Thompson, M. J., and Toomre, J.: 2000, Science 287, 2456.

Hoyt, D. V. and Schatten, K. H.: 1997, The Role of the Sun in Climate Change, Oxford University Press, New York, 279 pp.

Hoyt, D. V. and Schatten, K. H.: 1998a, Solar Phys. 179, 189.

Hoyt, D. V. and Schatten, K. H.: 1998b, Solar Phys. 181, 491.

Javaraiah, J. and Gokhale, M. H.: 1997, Solar Phys. 170, 389.

Kiepenheuer, K. O.: 1953, in G. P. Kuiper (ed.), The Sun, The University of Chicago Press, Chicago, p. 322.

Krivova, N. A., Solanki, S. K., and Beer, J.: 2002, Astron. Astrophys. 396, 235.

Lean, J.: 1990, Astrophys. J. 363, 718.

Lean, J., Beer, J., and Bradley, R.: 1995, Geophys. Res. Lett. 22, 3195.

Maunder, E. W.: 1890, Mon. Not. R. Astron. Soc. 50, 251.

Maunder, E. W.: 1904, Mon. Not. R. Astron. Soc. 64, 747.

McKinnon, J. A.: 1987, Rep. UAG-95, World Data Ctr. A for Solar-Terr. Phys., Boulder, CO, 112 pp. Mikhailutsa, V. P. and Makarova, V. V.: 1994, Solar Phys. 155, 391.

Moss, D.: 2004, Mon. Not. R. Astron. Soc. 352, L17.

Mursula, K., Usoskin, I. G., and Kovaltsov, G. A.: 2001, Solar Phys. 198, 51.

Mursula, K., Zieger, B., and Vilppola, J. H.: 2003, Solar Phys. 212, 201.

Nandy, D. and Choudhuri, A. R.: 2002, Science 296, 1671.

Ogurtsov, M. G., Nagovitsyn, Yu. A., Kocharov, G. E., and Jungner, H.: 2002, Solar Phys. 211, 371.

Rabin, D., Wilson, R. M., and Moore, R. L.: 1986, Geophys. Res. Lett. 13, 352.

Reid, G. C.: 1987, Nature 329, 142.

Rieger, E., Share, G. H., Forrest, D. J., Kanbach, G., Reppin, C., and Chupp, E. L.: 1984, Nature 312, 623.

Rozelot, J. P.: 1994, Solar Phys. 149, 149.

Sawyer, C.: 1968, Ann. Rev. Astron. Astrophys. 6, 115.

Schwabe, H.: 1844, Astron. Nachr. 21(495), 233.

Solanki, S. K., Krivova, N. A., Schüssler, M., and Fligge, M.: 2002, Astron. Astrophys. 396, 1029. 
Usoskin, I. G., Mursula, K., and Kovaltsov, G. A.: 2001, Astron. Astrophys. 370, L31.

Usoskin, I. G., Mursula, K., and Kovaltsov, G. A.: 2002, Geophys. Res. Lett. 29, 36.

Usoskin, I. G., Mursula, K., and Kovaltsov, G. A.: 2003, Astron. Astrophys. 403, 743.

Waldmeier, M.: 1935, Astron. Mitt. Zurich 14(133), 105-130.

Waldmeier, M.: 1939, Astron. Mitt. Zurich 14(138), 439 and 470.

Waldmeier, M.: 1961, The Sunspot-Activity in the Years 1610-1960, Schulthess, Zürich, Switzerland, $171 \mathrm{pp}$.

Waldmeier, M.: 1973, Solar Phys. 28, 389.

Wilson, R. M.: 1986, Solar Phys. 106, 29.

Wilson, R. M.: 1987, J. Geophys. Res. 92, 10,101.

Wilson, R. M.: 1988, Solar Phys. 115, 397. 